OPEN ACCESS

Edited by:

Feng Tao,

Texas A\&M University, United States

Reviewed by:

R. R. Ji,

Duke University, United States

Guang-Yin Xu,

Soochow University, China

${ }^{*}$ Correspondence:

Ke Ren

kren@umaryland.edu

Received: 03 August 2018 Accepted: 26 September 2018 Published: 16 October 2018

Citation:

Guo W, Imai S, Yang J-L, Zou S, Li H, Xu H, Moudgil KD, Dubner R, Wei F and Ren K (2018) NF-KappaB Pathway Is Involved in Bone Marrow Stromal Cell-Produced Pain Relief. Front. Integr. Neurosci. 12:49. doi: 10.3389/fnint.2018.00049

\section{NF-KappaB Pathway Is Involved in Bone Marrow Stromal Cell-Produced Pain Relief}

\author{
Wei Guo ${ }^{1}$, Satoshi Imai ${ }^{1,2}$, Jia-Le Yang ${ }^{1}$, Shiping Zou ${ }^{1}$, Huijuan $L i^{1,3}$, Huakun $X u^{4}$, \\ Kamal D. Moudgil ${ }^{5}$, Ronald Dubner ${ }^{1}$, Feng Wei ${ }^{1}$ and Ke Ren ${ }^{1 *}$ \\ ${ }^{1}$ Department of Neural and Pain Sciences, School of Dentistry \& Program in Neuroscience, University of Maryland, \\ Baltimore, MD, United States, ${ }^{2}$ Department of Clinical Pharmacology \& Therapeutics, Kyoto University Hospital, Kyoto, \\ Japan, ${ }^{3}$ Department of Neurology, The 3rd Affiliated Hospital of Sun Yat-sen University, Guangzhou, China, ${ }^{4}$ Department \\ of Advanced Oral Sciences and Therapeutics, University of Maryland School of Dentistry, Baltimore, MD, United States, \\ ${ }^{5}$ Department of Microbiology \& Immunology, University of Maryland, Baltimore, MD, United States
}

Bone marrow stromal cells (BMSCs) produce long-lasting attenuation of pain hypersensitivity. This effect involves BMSC's ability to interact with the immune system and activation of the endogenous opioid receptors in the pain modulatory circuitry. The nuclear factor kappa B (NF-kB) protein complex is a key transcription factor that regulates gene expression involved in immunity. We tested the hypothesis that the NF-kB signaling plays a role in BMSC-induced pain relief. We focused on the rostral ventromedial medulla (RVM), a key structure in the descending pain modulatory pathway, that has been shown to play an important role in BMSC-produced antihyperalgesia. In Sprague-Dawley rats with a ligation injury of the masseter muscle tendon (TL), BMSCs (1.5 M/rat) from donor rats were infused i.v. at 1 week post-TL. P65 exhibited predominant neuronal localization in the RVM with scattered distribution in glial cells. At 1 week, but not 8 weeks after BMSC infusion, western blot and immunostaining showed that p65 of NF-кB was significantly increased in the RVM. Given that chemokine signaling is critical to BMSCs' pain-relieving effect, we further evaluated a role of chemokine signaling in p65 upregulation. Prior to infusion of BMSCs, we transduced BMSCs with CCl4 shRNA, incubated BMSCs with RS 102895, a CCR2b antagonist, or maraviroc, a CCR5 antagonist. The antagonism of chemokines significantly reduced BMSC-induced upregulation of p65, suggesting that upregulation of p65 was related to BMSCs' pain-relieving effect. We then tested the effect of a selective NF-кB activation inhibitor, BAY 11-7082. The mechanical hyperalgesia of the rat was assessed with the von Frey method. In the pre-treatment experiment, BAY 11-7082 (2.5 and 25 pmol) was injected into the RVM at $2 \mathrm{~h}$ prior to BMSC infusion. Pretreatment with BAY 11-7082 attenuated BMSCs' antihyperalgesia, but post-treatment at 5 weeks post-BMSC was not effective. On the contrary, in TL rats receiving BAY 11-7082 without BMSCs, TL-induced hyperalgesia was attenuated, consistent with dual roles of NF-kB in pain hypersensitivity and BMSC-produced pain relief. These results indicate that the NF-kB 
signaling pathway in the descending circuitry is involved in initiation of BMSC-produced behavioral antihyperalgesia.

Keywords: orofacial pain, tendon ligation, mesenchymal stromal cells, BAY 11-7082, chemokine, rostral ventromedial medulla

\section{INTRODUCTION}

Chronic orofacial pain affects approximately 20 percent of the population and is a major health problem (Isong et al., 2008; Hargreaves, 2011). The most common persistent orofacial pain condition, temporomandibular joint disorders, affects the musculoskeletal and joint tissues, is heterogeneous in origin, and often not successfully treated. Bone marrow stromal cells (BMSCs) are a major type of mesenchymal stem (stromal) cells that can differentiate into lineages of the mesenchyme such as osteoblasts, chondrocytes and adipocytes in vitro (Pittenger et al., 1999). One important property of BMSCs is their potential for immune regulation (Davies et al., 2017), which have attracted great interest in exploring their therapeutic use in a variety of disease conditions including chronic pain. Both preclinical and clinical studies have shown pain-relieving or antihyperalgesic effect of BMSCs (Black et al., 2007; Abrams et al., 2009; Guo et al., 2011, 2017; Siniscalco et al., 2011; Roh et al., 2013; Sacerdote et al., 2013; Franchi et al., 2014; Vickers et al., 2014; Chen et al., 2015; Pettine et al., 2015, 2016; Watanabe et al., 2015; Evangelista et al., 2018). In a rat model of myogenic orofacial pain involving the ligation injury of one tendon of the masseter muscle (Guo et al., 2010), we have shown that systemic infusion of BMSCs produced long-term attenuation of persistent pain in both male and female rats, indicated by inhibition of thermal and mechanical nociception and pain aversion (Guo et al., 2011, 2016). We further showed that BMSC-produced antihyperalgesia required their interactions with host immune cells and activation of mu opioid receptors (MOR) in the pain-modulatory circuitry (Guo et al., 2014, 2017). Our findings call attention on immune regulation as a mechanism of BMSCs' therapeutic effects.

The nuclear factor kappa B (NF- $\kappa \mathrm{B})$ protein complex is a transcription factor that is found in almost all cell types and controls the transcription of multiple genes involved in immunity (Bonizzi and Karin, 2004). NF-кB is a dynamic nuclear transcription factor that can be activated by a variety of stimuli. Mesenchymal stromal cells promote neuroprotection via NFmediated gene transcription (Walker et al., 2010). Interestingly, tumor necrosis factor (TNF) produces neuroprotection and stimulates MOR expression in neurons involving the NF- $\mathrm{KB}$ pathway (Tamatani et al., 1999; Kraus et al., 2003; Fang et al., 2018). The activation of NF- $\mathrm{kB}$ correlates with increased anti-inflammatory cytokine interleukin (IL)-10 in human whole blood cell culture (Al-Hanbali et al., 2009) and induces IL-10 expression in human monocytes (Pilette et al., 2010). We reason that the NF-KB-involved signaling may play a role in BMSC-induced pain relief. This hypothesis was tested in the present study. We focused on the central mechanisms involving rostral ventromedial medulla (RVM), a key structure in the descending pain modulatory pathway, that has been shown to play an important role in BMSC-produced antihyperalgesia (Guo et al., 2011, 2017). We showed that there was an upregulation of p65 of NF- $\mathrm{B}$ in the RVM after BMSC treatment, and injection of the NF- $\kappa B$ activation inhibitor into the RVM attenuated BMSC-produced antihyperalgesia.

\section{MATERIALS AND METHODS}

\section{Animals}

Male Sprague-Dawley rats, $\approx 8$-week old at the time of surgery, were used (Envigo-Harlan). Animals were housed on the 9th floor of the University of Maryland School of Dentistry. The facility is an approved, registered research site (USDA \#MD-R-118) and accredited by AAALAC. Animals were kept under controlled environment conditions $\left(\approx 22^{\circ} \mathrm{C}\right)$, relative humidity $40 \%-60 \%, 12 \mathrm{~h} / 12 \mathrm{~h}$ light-dark cycles, and food and water ad libitum. The animals' conditions were monitored continuously throughout the course of studies, which include body weight, grooming, locomotion, ambulant activity and condition of the wound. The behavioral studies involve stimulation that produces only momentary additional pain/or discomfort and the rats can escape from the stimuli at any time.

All surgical procedures were performed under pentobarbital sodium $(20-50 \mathrm{mg} / \mathrm{kg}$ i.p.) anesthesia. Ligation of the tendon (TL) of the anterior superficial part of the rat masseter muscle was achieved via an intraoral approach as described elsewhere (Guo et al., 2010). Briefly, on the left intraoral site, a 5-mm long incision was made posterior-anteriorly lateral to the gingivobuccal margin in the buccal mucosa, beginning immediately next to the first molar. The tendon of the anterior superficial part of the rat masseter muscle was gently freed and tied with two chromic gut (4.0) ligatures, 2-mm apart. Animals were randomly assigned to experimental groups. The number of animals per group was determined by our previous studies and a power analysis. All experiments were carried out in accordance with the National Institutes of Health (NIH) Guide for the Care and Use of Laboratory Animals (NIH Publications No. 80-23) and approved by the Institutional Animal Care and Use Committee, University of Maryland School of Dentistry/Medicine.

\section{BMSC Procedures}

BMSCs were obtained from donor rats as described (Shen et al., 2006; Guo et al., 2011). The rats were euthanized with $\mathrm{CO}_{2}$ and both ends of the tibiae, femurs and humerus were cut off by scissors. A syringe fitted with an 18-gauge needle was inserted into the shaft of the bone and bone marrow was flushed out with culture medium (alpha-modified Eagle medium, Gibco, Carlsbad, CA, USA; 10\% fetal bovine serum, Hyclone, Logan, UT, USA). The bone marrow was then mechanically dissociated 

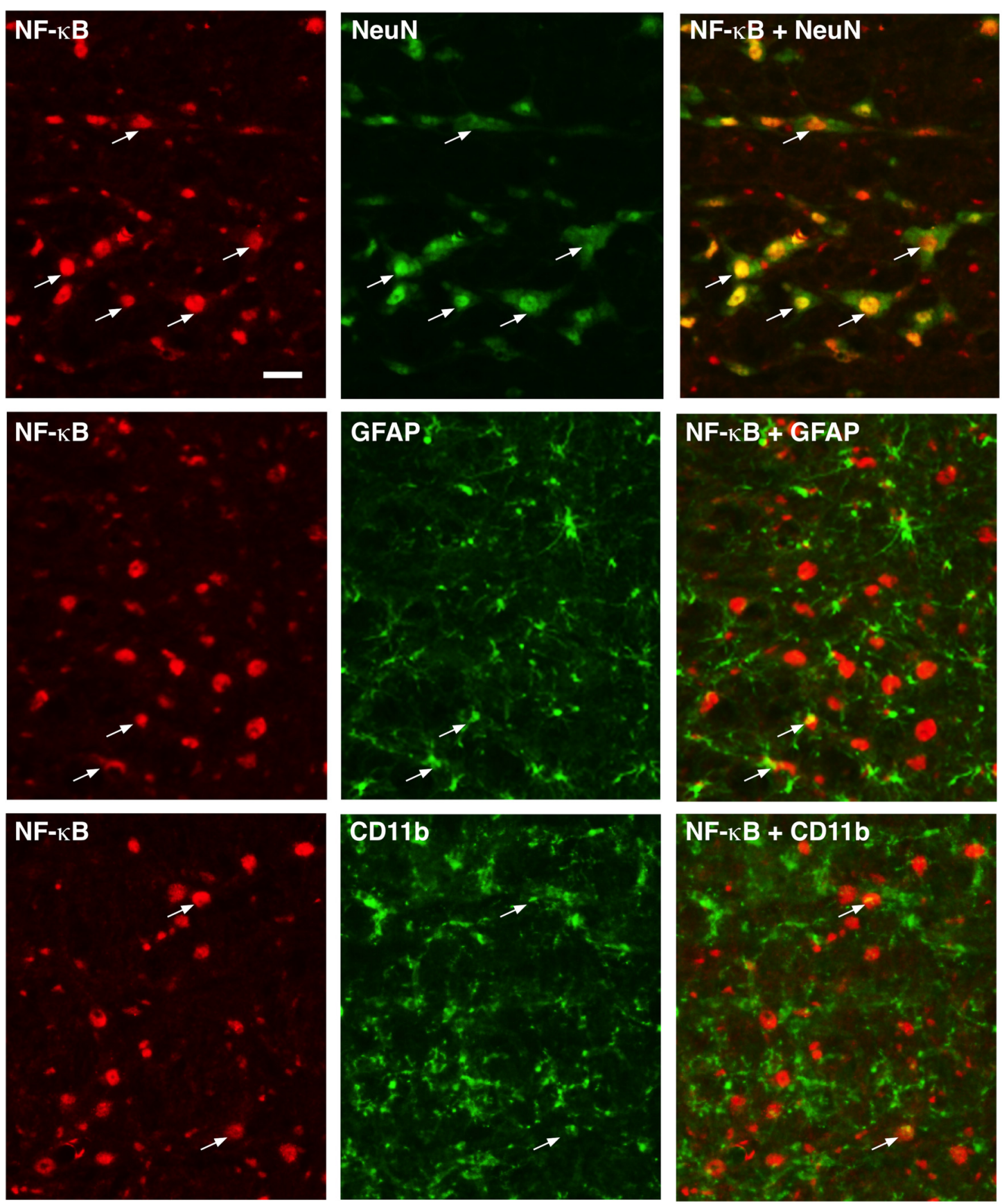

FIGURE 1 | Localization of p65 of nuclear factor kappa B (NF-kB) in rostral ventromedial medulla (RVM). Numerous neurons (top row) exhibited

p65 immunoreactivity shown by double immunofluorescence with NeuN. Few double-labeling profiles of p65 were shown with glial fibrillary acidic protein (GFAP; middle row; astrocytes) and CD11b (bottom row; microglia). Examples of double labeled profiles are indicated by arrows. Scale $=40 \mu \mathrm{m}$.

and the suspension passed through a $100-\mu \mathrm{m}$ cell strainer to remove debris. The cells were incubated at $37^{\circ} \mathrm{C}$ in $5 \% \mathrm{CO}_{2}$ in tissue-culture flasks $(100 \times 200 \mathrm{~mm}$; Sarstedt, Nümbrecht, Germany), and non-adherent cells removed by replacing the medium. At day 7, when the cultures reached $80 \%$ confluence, the cells were washed with PBS and harvested. The cell numbers were calculated by the Hemocytometer. For intravenous administration, $1.5 \times 10^{6}$ cells $(1.5 \mathrm{M})$ in $0.2 \mathrm{ml}$ PBS were slowly injected into one tail vein of the anesthetized rat over a 2 -min period using a 22-gauge needle. The property of expanded cells was assessed by flow cytometry with conventional markers (Guo et al., 2011). Flow cytometry analyses were performed at the University of Maryland GreenBaum Cancer Center Shared Flow Cytometry Facility. 

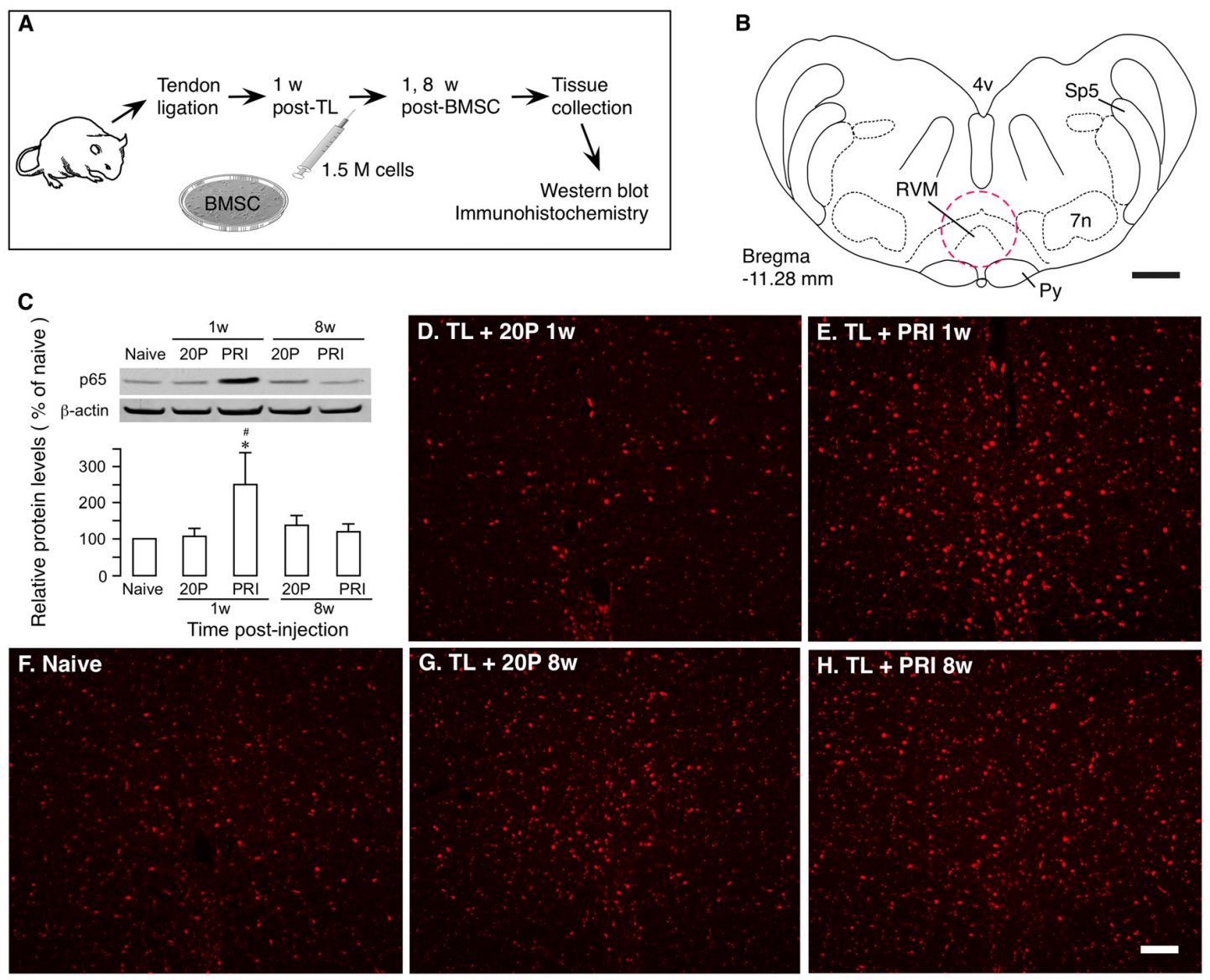

FIGURE 2 | Upregulation of NF-KB by bone marrow stromal cells (BMSCs). (A) Flowchart of the experiment. (B) A drawing of brainstem transverse section illustrating RVM (Paxinos and Watson, 2014). Dashed circle indicates the area punched for analysis. 4v, fourth ventricle; 7n, facial nucleus; Py, pyramidal tract; RVM, rostral ventromedial medulla; Sp5, spinal trigeminal tract. Scale $=1 \mathrm{~mm}$. (C) Effect of BMSCs on p65 of nuclear factor kappa B (NF-kB) in RVM. RVM tissues were collected at 1 week and 8 weeks after the BMSC injection. Total proteins isolated and separated. An example of the blot is shown on top and the relative protein levels are shown in the bottom histogram. $\beta$-actin was a loading control. p65 of NF-kB was upregulated at 1 week after injection of primary (PRI) BMSC. ${ }^{*} p<0.05$ vs. Naïv; $" \#<0.05$ vs. 20P (20-passage) BMSCs. (D-H) Immunostaining of p65 in RVM. Note an apparent increase in immunoreactivity in TL + PRI 1 week. (E) Scale $=0.1 \mathrm{~mm}$.

\section{Behavioral Testing}

All behavioral tests were conducted under blind conditions. Mechanical sensitivity of the orofacial region was assessed as described (Ren, 1999; Guo et al., 2010). A series of calibrated von Frey filaments were applied to the skin above the injured tendon or the corresponding contralateral side. An active withdrawal of the head from the probing filament was defined as a response. Each von Frey filament was applied five times at intervals of $5-10 \mathrm{~s}$. The response frequencies [(number of responses/number of stimuli) $\times 100 \%$ ] to a range of von Frey filament forces were determined and a stimulus-response frequency (S-R) curve plotted. After a non-linear regression analysis, an $\mathrm{EF}_{50}$ value, defined as the effective von Frey filament force $(\mathrm{g})$ that produces a $50 \%$ response frequency, was derived from the S-R curve (Prism, GraphPad; Guo et al., 2004). A leftward shift of the $\mathrm{S}-\mathrm{R}$ curve, resulting in a reduction of $\mathrm{EF}_{50}$, occurred after TL, which suggests the development of mechanical hypersensitivity, including allodynia and hyperalgesia.

\section{Western Blot}

Rats were anesthetized with isoflurane (3\%) and quickly decapitated. The brainstem tissue block that included the RVM was harvested by taking punches with a 15-gauge needle. The tissues were homogenized in solubilization buffer $(50 \mathrm{mM}$ Tris.HCl, pH 8.0; 150 mM NaCl, 1 mM EDTA, 1\% NP40, 0.5\% deoxycholic acid, $0.1 \%$ SDS, $1 \mathrm{mM} \mathrm{Na} \mathrm{VO}_{4}, 1 \mathrm{U} / \mathrm{ml}$ aprotinin, $20 \mu \mathrm{g} / \mathrm{ml}$ leupeptin, $20 \mu \mathrm{g} / \mathrm{ml}$ pepstatin A). The homogenate was centrifuged at $20,200 \times g$ for $10 \mathrm{~min}$ at $4^{\circ} \mathrm{C}$. The supernatant was removed. The protein concentration was determined using a detergent-compatible protein assay with a bovine serum albumin standard. For detecting the immunoreactivity with near-infrared fluorescence using the Odyssey Infrared Imaging System (Odyssey ${ }^{\circledR}$ CLx, LI-COR, Lincoln, NE, USA), 50- $\mu \mathrm{g}$ protein samples were denatured by boiling for $5 \mathrm{~min}$ and loaded onto 4\%-20\% Bis-Tris gels (Invitrogen). After electrophoresis, proteins were transferred to nitrocellulose membranes. The membranes were blocked for $1 \mathrm{~h}$ with Odyssey Blocking Buffer 
and then incubated with primary antibodies (Anti-NF- $\kappa$ B, p65 subunit (Cell Signaling) diluted in Odyssey Blocking Buffer at $4^{\circ} \mathrm{C}$ overnight, followed by washing with PBS containing $0.1 \%$ Tween 20 (PBST) three times. The membranes were then incubated for $1 \mathrm{~h}$ with IRDye800CW-conjugated goat anti-rabbit IgG and IRDye680-conjugated goat anti-mouse IgG secondary antibodies (LI-COR) diluted in Odyssey Blocking Buffer. The blots were further washed three times with PBST and rinsed with PBS. Proteins were visualized by scanning the membrane with 700 and $800-\mathrm{nm}$ channels. The loading and blotting of the amount of protein was verified by reprobing the membrane with anti- $\beta$-actin and with Coomassie blue staining.

\section{Immunohistochemistry}

Rats were deeply anesthetized with pentobarbital sodium (100 mg/kg, i.p.) and perfused transcardially with $4 \%$ paraformaldehyde in $0.1 \mathrm{M}$ phosphate buffer at $\mathrm{pH}$ 7.4. The brain stem was removed, post-fixed, and transferred to $25 \%$ sucrose $(\mathrm{w} / \mathrm{v})$ for cryoprotection. Transverse sections $(20-\mu \mathrm{m})$ were cut with a cryostat. The free-floating sections were incubated with relevant antibodies with $1 \%$ normal goat sera and $0.3 \%$ Triton $\mathrm{X}-100$ overnight at $4^{\circ} \mathrm{C}$. After washes in PBS, the sections were incubated with relevant IgGs conjugated to Cy3 or Cy2 (1:500; Jackson ImmunoResearch, West Grove, PA, USA) for $4 \mathrm{~h}$ at room temperature or overnight at $4^{\circ} \mathrm{C}$. Double immunofluorescent staining was performed for p65 with NeuN (Chemicon), glial fibrillary acidic protein (GFAP; Chemicon) or CD11b (AbD Serotec, Novus). Following washes, the stained sections were mounted on gelatin-coated slides and coverslipped with Vectashield (Vector Laboratories). Slides were examined with a Nikon fluorescence microscope and images were captured with a CCD Spot camera. Control sections were processed with the same method except that the primary antisera are omitted.

\section{Brainstem Microinjections}

Rats were anesthetized with $2 \%-3 \%$ isoflurane in a gas mixture of $30 \% \mathrm{O}_{2}$ balanced with $70 \%$ nitrogen and placed in a Kopf stereotaxic instrument (Kopf Instruments, Tujunga, CA, USA). A midline incision was made after infiltration of lidocaine (2\%) into the skin. A midline opening was made in the skull with a dental drill for inserting an injection needle into the target site. The coordinates for the RVM were: $10.5-11.5 \mathrm{~mm}$ caudal to the Bregma, midline and $9.0 \mathrm{~mm}$ ventral to the surface of the cerebellum (Paxinos and Watson, 2014). Microinjections were performed by delivering drug solutions slowly over a 10-min period using a $500 \mathrm{nl}$ Hamilton syringe with a 32-gauge needle. The injection needle was left in place for at least $15 \mathrm{~min}$ before being slowly withdrawn. The wound was closed and animals were returned to their cages after recovering from anesthesia. For histology verification of the injection site, $30-\mu \mathrm{M}$ coronal brainstem sections were stained with Neurotrace ${ }^{\mathrm{TM}}$ 500/525 Green fluorescent Nissl Stain (Invitrogen; 1:500 for $20 \mathrm{~min})$.

\section{RNAi}

Ccl4 shRNA (Accession Number NM_053858.1 CDS, target sequence: 90: TCCCACTTCCTGCTGCTTCTCTTACACCT) was transduced into cultured BMSCs [Ccl4 RNAi lentivirus (piLenti-siRNA-GFP, abm ${ }^{\circledR}$ Richmond, BC, Canada)]. BMSCs were plated onto $10-\mathrm{cm}$ plate before transduction. When they reached $80 \%$ confluence $5 \mathrm{ml}$ medium (without serum) was added with $4 \mu \mathrm{l}$ polybrene $(8 \mu \mathrm{g} / \mathrm{ml})$ and $50 \mu \mathrm{l}$ of ViralPlus Transduction Enhancer G698 (abm). Eighty-microliter Ccl4 shRNA lentivirus or control shRNA lentivirus was then added to the plate. Cells were incubated at $37^{\circ} \mathrm{C}$ with $5 \% \mathrm{CO}_{2}$ and collected at $72 \mathrm{~h}$ following transduction. Successful transduction and knockdown of Ccl4 were verified (Guo et al., 2017).

\section{Drugs}

All drugs were purchased: NF- $\mathrm{B}$ activation inhibitor BAY 117082 [(E)-3-(4-Methylphenylsulfonyl)-2-propenenitrile] MW 207.25, CAS Number 19542-67-7 (Calbiochem), CCR2 receptor antagonist RS-102895 hydrochloride $\left\{1^{\prime}-[2-[4-\right.$ (Trifluoromethyl)phenyl]ethyl]-spiro[4H-3,1-benzoxazine-4,4' peperidin]-2(1H)-one\} MW 426.86, CAS number 300815-41-2 (Sigma-Aldrich), and CCR5 antagonist maraviroc $\{4,4-$ Difluoro-N-[(1S)-3-[(3-exo)-3-[3-methyl-5-(1-methylethyl)-4H -1,2,4-triazol-4-yl]-8-azabicyclo[3.2.1] oct-8-yl]-1-phenylpropyl] cyclohexanecarboxamide\} MW 513.68, CAS Number 37634865-1 (R\&D). Drugs were dissolved in 5\% dimethyl sulfoxide and saline.

\section{Data Analysis}

Data are presented as means \pm SEM. Analysis of variance (ANOVA) and the post hoc Tukey test was performed for protein data. One- or two-way ANOVA with repeated measures was used for comparison of $\mathrm{EF}_{50} \mathrm{~s}$, followed by post hoc test with corrections for multiple comparisons. $P<0.05$ was considered significant for all cases.

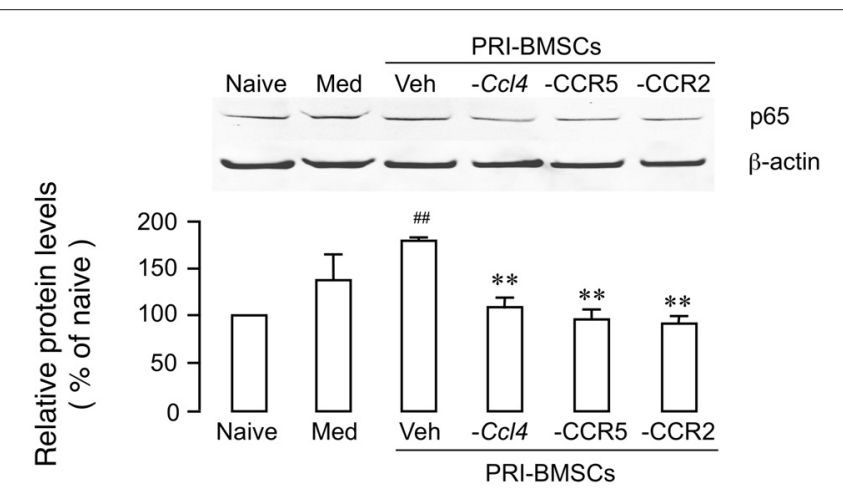

FIGURE 3 | Attenuation of BMSC-induced upregulation of p65 by antagonism of chemokine signaling associated with BMSCs' pain-relieving effect. Prior to infusion of BMSCs, Cc/4 was knocked down from primary BMSCs by transduction with CCl4 shRNA, CCR2 was blocked by pretreatment of BMSCs with RS 102895 (10 $\mu \mathrm{M}$ for 24 h), a CCR2b antagonist, and CCR5 was blocked by pretreatment of BMSCs with maraviroc (200 nM for $24 \mathrm{~h}$ ), a CCR5 antagonist. Culture medium (Med) was a control for BMSCs. ${ }^{\# \#} p<0.01$ vs. Naïve; ${ }^{* *} p<0.01$ vs. Veh. $N=4$. 


\section{A RVM injection}

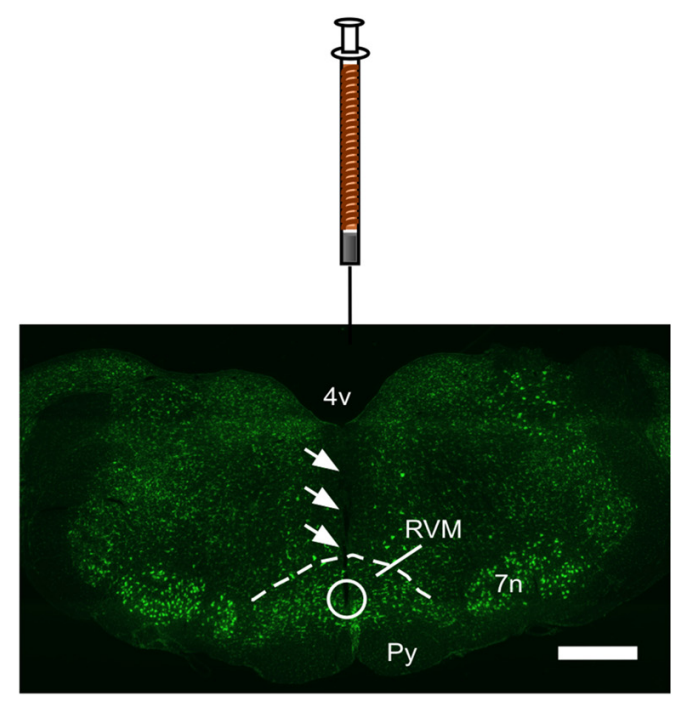

\section{Post-treatment}

$$
\begin{aligned}
& \square \operatorname{Veh}(n=6) \\
& \square \operatorname{BAY}(n=6)
\end{aligned}
$$

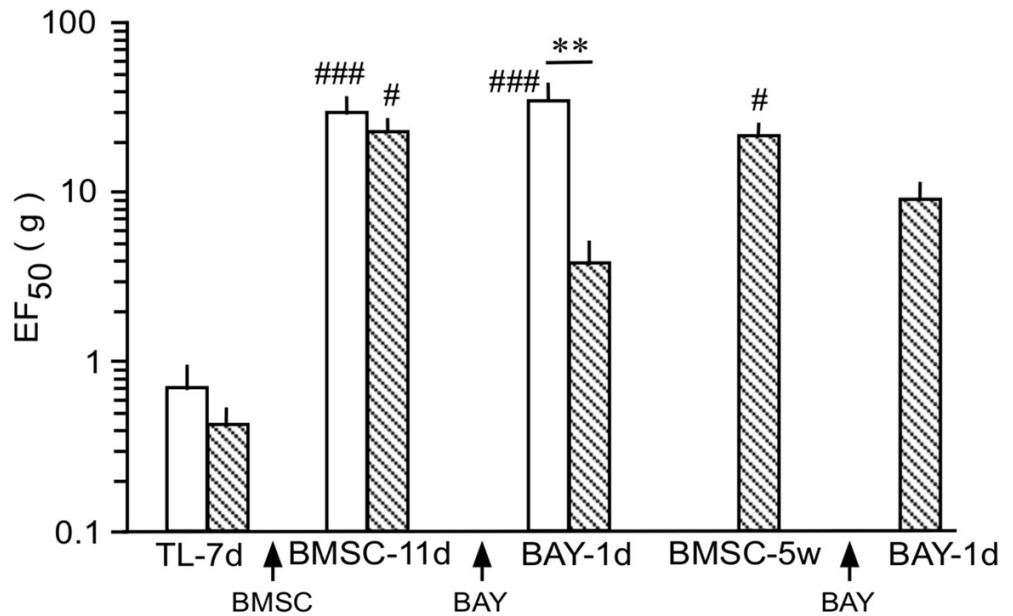

B Pre-treatment

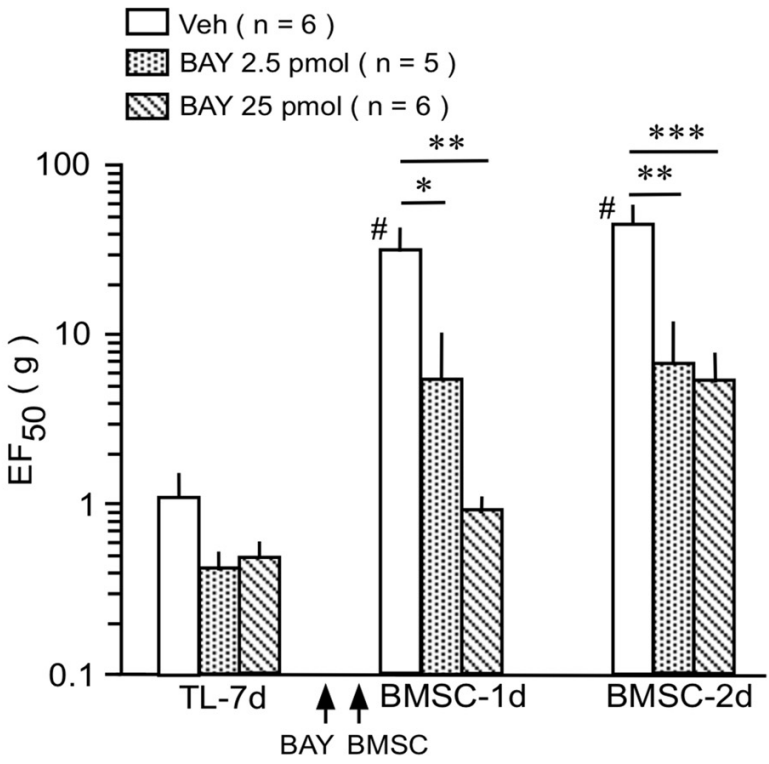

D Inhibitor only

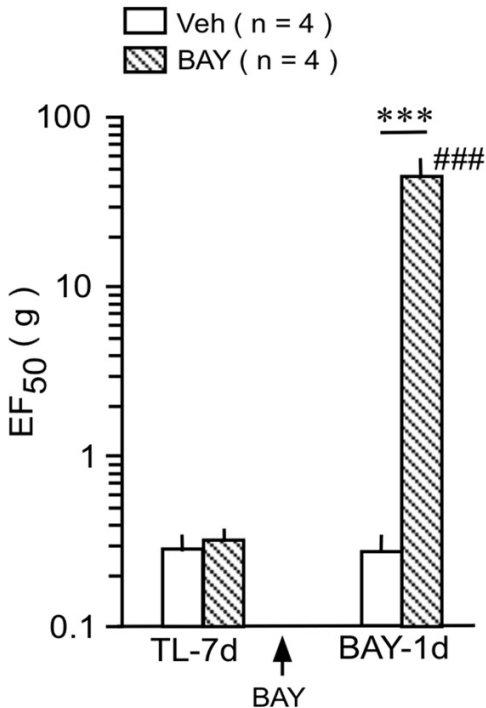

FIGURE 4 | Effect of NF-кB activation inhibitor on BMSC-produced antihyperalgesia. (A) Image shows the injection site in RVM. Coronal brainstem sections were stained with green fluorescent Nissl stain. Arrows indicate the injection needle track and circle shows the site of injection. Scale $=1 \mathrm{~mm}$. (B) Pre-treatment experiment, BAY 11-7082 (BAY) was injected at $2 \mathrm{~h}$ prior to BMSC infusion. (C) Post-treatment experiment, BAY was injected at 11 days and 5 weeks after BMSC infusion. (D) Inhibitor only experiment, BAY was injected at 7 days post-TL (TL-7d) and BMSCs were not infused. ${ }^{*} p<0.05,{ }^{* *} p<0.01$, ${ }^{* * *} p<0.001$ vs. veh; \#p<0.05, \#\# $p<0.001$ vs. TL-7d.

\section{RESULTS}

NF- $\kappa \mathrm{B}$ functions as dimers formed from five-member proteins (p50, p52, p65, RelB and c-Rel; Bonizzi and Karin, 2004). The p50/p65 dimer is the most abundant and considered canonical. P50 contains a nuclear localization sequence, provides DNA binding, and interacts with the IкB
(NF-кB inhibitory proteins), while p65 has a transcription activation domain and is a transcriptional activator. Immunostaining of p65 showed wide-spread distribution in RVM tissues (Figure 1). Double immunofluorescence labeling indicated p65 expression in neurons, as shown by extensive co-localization with NeuN, a neuronal marker (Figure 1, Top row). In contrast, fewer scattered double-labeling 
profiles were seen with GFAP, an astrocyte marker (Figure 1, Middle row), and CD11b, a microglia marker (Figure 1, Bottom row).

Ligation injury of the masseter muscle tendon was produced to assess BMSC-produced effects as described previously (Guo et al., 2010, 2011). Masseter TL induces nociceptive hypersensitivity lasting for months. At 1 week after TL, $1.5 \times 10^{6}$ BMSCs were infused into one tail vein and at 1 and 8 weeks after BMSC infusion, RVM tissues were collected for western blot and immunohistochemistry (Figures 2A,B). We have shown that primary, but not 20-passage (20P) BMSCs, attenuate persistent pain hypersensitivity (Guo et al., 2011). Thus, 20P BMSCs were used as a control. Western blot showed that p65 of NF-кB was significantly upregulated at 1 week after injection of primary BMSCs, compared to naïve and 20P BMSC-treated TL rats (Figure 2C). There was no change in p65 at 8 weeks after BMSC infusion. Similar changes in p65 immunostaining were observed in RVM (Figures 2D-H).

We have shown previously that chemokine signaling is critical to BMSCs' pain-relieving effect (Guo et al., 2017). Compared to primary BMSCs, a number of chemokines/receptors were significantly down-regulated in 20P BMSCs including CCL4, CCR2 and CCR5, which underlies their inability to produce antihyperalgesia. Knock-down of CCL4 from BMSCs or blockage of CCR2 or CCR5 reverse BMSC-produced upregulation of MOR and antihyperalgesia (Guo et al., 2017, 2018). To evaluate a role of chemokine signaling in p65 upregulation, we performed the same manipulations prior to infusion of BMSCs, including transduction of primary BMSCs with Ccl4 shRNA, incubation of primary BMSCs with RS $102895(10 \mu \mathrm{M}$ for $24 \mathrm{~h}$ ), a CCR2b antagonist, or maraviroc (200 nM for $24 \mathrm{~h}$ ), a CCR5 antagonist. The antagonism of chemokines significantly reduced BMSC-induced upregulation of p65 (Figure 3), suggesting that upregulation p65 was related to BMSCs' pain-relieving effect.

We then tested the effect of a selective NF- $\kappa \mathrm{B}$ activation inhibitor, BAY 11-7082. In rats receiving TL, mechanical sensitivity was assessed as described (Ren, 1999; Guo et al., 2010). In the pre-treatment experiment, BAY 11-7082 (2.5 and $25 \mathrm{pmol} / 0.5 \mu \mathrm{l})$ or drug vehicle $(0.5 \mu \mathrm{l})$ was injected at $2 \mathrm{~h}$ prior to BMSC infusion (Figures $4 \mathbf{A}, \mathbf{B}$ ). In vehicle-treated rats, $\mathrm{EF}_{50}$ was significantly increased following infusion of BMSCs, compared to 7 days post-TL (TL-7d) rats (Figure $4 \mathrm{~B} ; p<0.05$ ), indicating antihyperalgesia. Pretreatment with BAY 11-7082 significantly attenuated this antihyperalgesia $(p<0.05-0.001)$, examined at 1-2 days after BMSC infusion (Figure 4B). Post-treatment with BAY 11-7082 (25 pmol/0.5 $\mu \mathrm{l})$ at 11 days after BMSC infusion led to a smaller reduction of $\mathrm{EF}_{50}$, compared to the pretreatment (Figures 4B,C). Injection of BAY 11-7082 at 5 weeks post-BMSC did not have an effect on BMSC-induced antihyperalgesia (Figure 4C).

Although NF- $\kappa \mathrm{B}$ is seemingly involved in BMSC-induced protection as shown above, it is well known that activation of NF- $\kappa \mathrm{B}$ pathway contributes to pro-inflammatory responses, characterized by induction of pro-inflammatory cytokines such as IL-1 $\beta$ (Ghosh et al., 1998; Bonizzi and Karin,

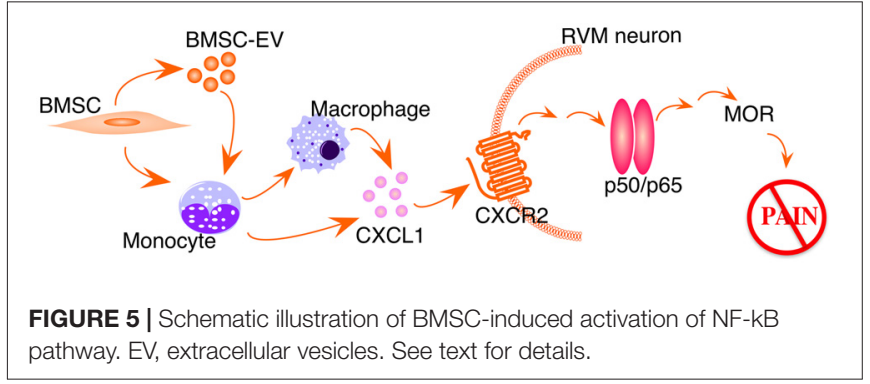

2004). Accordingly, NF-кB-involved signaling plays a role in enhanced pain sensitivity (Ross-Huot et al., 2013; Zhou et al., 2018). In our inhibitor only experiment, BAY 11-7082 (25 pmol/0.5 $\mu \mathrm{l})$ was injected into the RVM at 7 days after TL and BMSCs were not infused. BAY 11-7082 significantly raised $\mathrm{EF}_{50} \mathrm{~s}$ at 1 day (Figure 4D) and 2 days (not shown) after injection, indicating attenuation of TL-induced pain. This result is consistent with dual roles of NF- $\kappa \mathrm{B}$ in pain hypersensitivity and BMSC-produced pain relief.

\section{DISCUSSION}

Our recent results indicate that interactions between infused BMSCs and the host immune system underlie their antihyperalgesic effect (Guo et al., 2017). Through the monocyte/macrophage population and associated chemokines and their receptors, BMSCs upregulate MOR in the RVM and produce potent antihyperalgesia. To extend these findings, we show here that the NF- $\kappa \mathrm{B}$ pathway, a pivot of immune responses, is involved in BMSC's pain-relieving effect. In the context of TL-induced orofacial hyperalgesia and transplantation of BMSCs, p65 of NF- $\mathrm{B}$ was upregulated in the RVM and inhibition of NF- $\mathrm{B}$ rekindled hyperalgesia.

The significance of upregulation of p65 in BMSCs' antihyperalgesia is supported by its dependance on related chemokine signaling. Down regulation of CCL4 from BMSCs or blocking CCR2 and CCR5 of BMSCs prevented BMSCs from producing antihyperalgesia (Guo et al., 2017, 2018). Consistently, we show that p65 upregulation was significantly reduced under those conditions. However, the NF- $\kappa B$ pathway did not seem to be important for the late maintenance of pain-relieving effect of BMSCs. P65 was only upregulated at the first week after the BMSC treatment and pretreatment with NF-кB inhibitor was able to reverse antihyperalgesia. Contrastingly, post-treatment with BAY 11-7082 at the late 5 -week time point was ineffective. These observations suggest that NF- $\kappa \mathrm{B}$ activation is mainly involved in initialization of BMSCs' therapeutic effect.

The present findings represent an apparent paradox with regard to function of $N F-\kappa B$ signaling. It is well known that $\mathrm{NF} \kappa \mathrm{B}$ activation is pro-inflammatory, characterized by induction of pro-inflammatory cytokines (Ghosh et al., 1998; Bonizzi and Karin, 2004). Studies have shown contribution of NF- $\kappa$ B to persistent pain (de Mos et al., 2009; Ross-Huot et al., 2013; 
Borghi et al., 2017). Thus, under persistent pain conditions without BMSC treatment, suppressing NF- $\kappa$ B should attenuate hyperalgesia (Ledeboer et al., 2005; Zhou et al., 2018). Without BMSCs, our results are consistent with pronociceptive role of NF- $\kappa$ B, as BAY 11-7082 attenuated mechanical hypersensitivity in TL rats.

It is appreciated that the function of $N F-\kappa B$ is diverse and dual roles of $\mathrm{NF}-\kappa \mathrm{B}$ signaling have been noticed. In fact, genes encoding proteins with opposite functions can

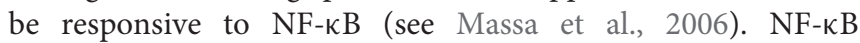
activity is also involved in the resolution of inflammation (Lawrence et al., 2001). Both pro- and anti-inflammatory cytokines can be induced by NF-kB activation (see Chang and Vancurova, 2013). We have observed upregulation of IL-10 and CD206, a marker of anti-inflammatory microglia, in the RVM after BMSC injection in TL rats (Ren, in press), which correlates with the increase of $\mathrm{p} 65$. NF- $\mathrm{B}$ has been shown to positively regulate Foxp3 expression in Tregs ( $\mathrm{T}$ regulatory cells; Long et al., 2009), which is in line with its role in the resolution of inflammation. RelB of NF- $\kappa$ B can induce and suppress gene expression (Madge and May, 2011). In the multiple sclerosis model, the activation of NF- $\mathrm{B}$ protects oligodendroctyes against inflammation (Stone et al., 2017). The antihyperalgesic effects of NF- $\mathrm{B}$ could be explained by phenotype switching action of BMSCs on immune cells. BMSCs can reprogram monocyte/macrophage and promote an anti-inflammatory phenotype (Al-Hanbali et al., 2009; Németh et al., 2009; Dayan et al., 2011; Guo et al., 2017), likely involving NF- $\kappa$ B activation (Pilette et al., 2010). Together with endogenous opioids, BMSC-produced antihyperalgesia should offset the pain facilitatory effect of NF- $\mathrm{NB}$.

We noted that p65 of NF- $\mathrm{B}$ widely distributed in RVM neurons, which provides an integrated platform for interactions between the neuron and immune system. We have shown that RVM MOR-containing neurons contribute to BMSC-produced antihyperalgesia (Guo et al., 2011, 2017). MOR activates NF-кB signaling (Hou et al., 1996; Wang et al., 2004; Liu and Wong, 2005). Upregulation of MOR expression in neurons may also be downstream to NF- $\mathrm{B}$ activation (Kraus et al., 2003; Wei and Loh, 2011; Wagley et al., 2013). In cell cultures, TNF stimulates MOR expression in immune cells as well as neurons involving the NF- $\kappa$ B pathway (Kraus et al., 2003). Additionally, through the NF- $\kappa \mathrm{B}$ pathway, TNF promoted IL-1receptor antagonist release from mesenchymal stromal cells to facilitate wound healing (Kou et al., 2018). Interestingly, compared to primary BMSCs, TNF is dramatically down-regulated in 20P BMSCs that are ineffective in producing antihyperalgesia (Guo et al., 2017) and TNF activate NF- $\kappa$ B pathway through phosphorylation of p65 in HeLa cell cultures (Wang and Baldwin, 1998). On the other hand, IL-6 induces MOR transcription in the human neuroblastoma cell line that involves transcription factors signal transducers and activators of transcription 1 (STAT1) and STAT3, but not NF- $\kappa$ B (Börner et al., 2004). It would be interesting to know cytokine-specific NF-кB-mediated regulation of MOR in vivo after transplantation of BMSCs.

Studies have shown that CXCL1-CXCR2 chemokine signaling produces biological effects involving downstream NF- activation (Cai et al., 2010; Dong et al., 2013). We speculate that CXCL1-CXCR2 signaling induces NF- $\mathrm{B}$ upregulation in our model (Figure 5). Systemic BMSCs interact directly, or via their secreted extracellular vesicles, with monocytes in the circulation. Removing monocytes/macrophages largely attenuates BMSCs' antihyperalgesia (Guo et al., 2017) and monocytes have been shown as major recipient of BMSC-derived extracellular vesicles (Di Trapani et al., 2016). In TL rats receiving BMSC treatment, CXCL1 is specifically upregulated in peripheral monocytes and cerebrospinal fluid, and CXCR2 is upregulated in RVM neurons containing MOR (Guo et al., 2017). Further, the CXCL1-CXCR2 signaling in RVM neurons is required for BMSC-produced antihyperalgesia (Guo et al., 2017). It remains to be shown that BMSC-induced upregulation of MOR is causally related to NF- $\kappa \mathrm{B}$ activation involving the CXCL1-CXCR2 axis.

The present results extend our previous findings that in vivo immune interactions underlie mechanisms of BMSC-produced pain relief. We provide first evidence that the NF- $\kappa$ B signaling pathway in the descending circuitry is involved in BMSC-produced behavioral antihyperalgesia. The activation of NF- $\kappa \mathrm{B}$ following BMSCs leads to a protective outcome that opposes to a conventional proinflammatory role. The results suggest that activation of NF- $\mathrm{B}$ may have diverse functional consequences, depending upon the type of stimuli and cellular environment. Future studies should explore cell-specific involvement of different NF- $\kappa B$ dimers, canonical and non-canonical (Massa et al., 2006), under different pathological and therapeutic conditions.

\section{AUTHOR CONTRIBUTIONS}

WG: conception and design, collection and assembly of behavioral and western blot data, data analysis and interpretation, manuscript writing. SI: conception and design, BMSC cultures, data analysis and interpretation, manuscript writing. J-LY: collection and assembly of immunohistochemistry data. SZ: collection and assembly of behavioral data. HL: collection and assembly of western blot data. HX: BMSC procedure and design. KM: design and data analysis. RD: conception and design, data analysis and interpretation, manuscript writing. FW: conception and design, data analysis and interpretation, manuscript writing. KR: conception and design, assembly of data, data analysis and interpretation, manuscript writing. All authors gave final approval of the manuscript.

\section{FUNDING}

This work was supported by the Maryland Stem Cell Foundation grant 2014-MSCRFI-0584 (KR); NIH grants: DE025137 (KR), NS019296 (FW), DE021804 (RD); The Team-Building Project for Stem Cell Research (K00008), SYSU (HL).

\section{ACKNOWLEDGMENTS}

We thank Dr. George T.-J. Huang for his advice on the early stages of this project. 


\section{REFERENCES}

Abrams, M. B., Dominguez, C., Pernold, K., Reger, R., Wiesenfeld-Hallin, Z., Olson, L., et al. (2009). Multipotent mesenchymal stromal cells attenuate chronic inflammation and injury-induced sensitivity to mechanical stimuli in experimental spinal cord injury. Restor. Neurol. Neurosci. 27, 307-321. doi: 10.3233/RNN-2009-0480

Al-Hanbali, M., Ali, D., Bustami, M., Abdel-Malek, S., Al-Hanbali, R., Alhussainy, T., et al. (2009). Epicatechin suppresses IL-6, IL-8 and enhances IL-10 production with NF- $\mathrm{B}$ nuclear translocation in whole blood stimulated system. Neuro. Endocrinol. Lett. 30, 131-138.

Black, L. L., Gaynor, J., Gahring, D., Adams, C., Aron, D., Harman, S., et al. (2007). Effect of adipose-derived mesenchymal stem and regenerative cells on lameness in dogs with chronic osteoarthritis of the coxofemoral joints: a randomized, double-blinded, multicenter, controlled trial. Vet. Ther. 8, 272-284.

Bonizzi, G., and Karin, M. (2004). The two NF-кB activation pathways and their role in innate and adaptive immunity. Trends Immunol. 25, 280-288. doi: 10.1016/j.it.2004.03.008

Borghi, S. M., Fattori, V., Ruiz-Miyazawa, K. W., Miranda-Sapla, M. M., Casagrande, R., Pinge-Filho, P., et al. (2017). Leishmania (L). amazonensis induces hyperalgesia in balb/c mice: contribution of endogenous spinal cord TNF $\alpha$ and NFкB activation. Chem. Biol. Interact. 268, 1-12. doi: 10.1016/j.cbi. 2017.02.009

Börner, C., Kraus, J., Schröder, H., Ammer, H., and Höllt, V. (2004). Transcriptional regulation of the human $\mu$-opioid receptor gene by interleukin-6. Mol. Pharmacol. 66, 1719-1726. doi: 10.1124/mol.104.003806

Cai, S., Batra, S., Lira, S. A., Kolls, J. K., and Jeyaseelan, S. (2010). CXCL1 regulates pulmonary host defense to Klebsiella Infection via CXCL2, CXCL5, NF-кB and MAPKs. J. Immunol. 185, 6214-6225. doi: 10.4049/jimmunol.0903843

Chang, T.-P., and Vancurova, I. (2013). NFкB function and regulation in cutaneous T-cell lymphoma. Am. J. Cancer Res. 3, 433-445.

Chen, G., Park, C.-K., Xie, R.-G., and Ji, R.-R. (2015). Intrathecal bone marrow stromal cells inhibit neuropathic pain via TGF- $\beta$ secretion. J. Clin. Invest. 125, 3226-3240. doi: 10.1172/jci80883

Davies, L. C., Heldring, N., Kadri, N., and Le Blanc, K. (2017). Mesenchymal stromal cell secretion of programmed death-1 ligands regulates $\mathrm{T}$ cell mediated immunosuppression. Stem Cells 35, 766-776. doi: 10.1002/stem.2509

Dayan, V., Yannarelli, G., Billia, F., Filomeno, P., Wang, X. H., Davies, J. E., et al. (2011). Mesenchymal stromal cells mediate a switch to alternatively activated monocytes/macrophages after acute myocardial infarction. Basic Res. Cardiol. 106, 1299-1310. doi: 10.1007/s00395-011-0221-9

de Mos, M., Laferrière, A., Millecamps, M., Pilkington, M., Sturkenboom, M. C., Huygen, F. J., et al. (2009). Role of NFкB in an animal model of complex regional pain syndrome-type I (CRPS-I). J. Pain. 10, 1161-1169. doi: 10.1016/j. jpain.2009.04.012

Di Trapani, M., Bassi, G., Midolo, M., Gatti, A., Kamga, P. T., Cassaro, A., et al. (2016). Differential and transferable modulatory effects of mesenchymal stromal cell-derived extracellular vesicles on T, B and NK cell functions. Sci. Rep. 6:24120. doi: 10.1038/srep24120

Dong, Y. L., Kabir, S. M., Lee, E. S., and Son, D. S. (2013). CXCR2-driven ovarian cancer progression involves upregulation of proinflammatory chemokines by potentiating NF-кB activation via EGFR-transactivated Akt signaling. PLoS One 8:e83789. doi: 10.1371/journal.pone.0083789

Evangelista, A. F., Vannier-Santos, M. A., de Assis Silva, G. S., Silva, D. N., Juiz, P. J. L., Nonaka, C. K. V., et al. (2018). Bone marrow-derived mesenchymal stem/stromal cells reverse the sensorial diabetic neuropathy via modulation of spinal neuroinflammatory cascades. J. Neuroinflammation 15:189. doi: 10.1186/s12974-018-1224-3

Fang, C., Xie, L., Liu, C., Fu, C., Ye, W., Liu, H., et al. (2018). Tanshinone IIA improves hypoxic ischemic encephalopathy through TLR-4-mediated NF-кB signal pathway. Mol. Med. Rep. 18, 1899-1908. doi: 10.3892/mmr. 2018.9227

Franchi, S., Castelli, M., Amodeo, G., Niada, S., Ferrari, D., Vescovi, A., et al. (2014). Adult stem cell as new advanced therapy for experimental neuropathic pain treatment. Biomed Res. Int. 2014:470983. doi: 10.1155/2014/470983

Ghosh, S., May, M. J., and Kopp, E. B. (1998). NF-кB and Rel proteins: evolutionarily conserved mediators of immune responses. Annu. Rev. Immunol. 16, 225-260. doi: 10.1146/annurev.immunol.16.1.225
Guo, W., Chu, Y.-X., Imai, S., Yang, J.-L., Zou, S., Mohammad, Z., et al. (2016). Further observations on the behavioral and neural effects of bone marrow stromal cells in rodent pain models. Mol. Pain 12:1744806916658043. doi: $10.1177 / 1744806916658043$

Guo, W., Imai, S., Dubner, R., and Ren, K. (2014). Multipotent stromal cells for arthritic joint pain therapy and beyond. Pain Manag. 4, 153-162. doi: $10.2217 / \mathrm{pmt} .14 .1$

Guo, W., Imai, S., Yang, J.-L., Zou, S., Watanabe, M., Chu, Y.-X., et al. (2017). In vivo immune interactions of multipotent stromal cells underlie their long-lasting pain-relieving effect. Sci. Rep. 7:10107. doi: 10.1038/s41598-01710251-y

Guo, W., Wang, H., Zou, S., Gu, M., Watanabe, M., Wei, F., et al. (2011). Bone marrow stromal cells produce long-term pain relief in rat models of persistent pain. Stem Cells 29, 1294-1303. doi: 10.1002/stem.667

Guo, W., Wang, H., Zou, S., Wei, F., Dubner, R., and Ren, K. (2010). Long lasting pain hypersensitivity following ligation of the tendon of the masseter muscle in rats: a model of myogenic orofacial pain. Mol. Pain 6:40. doi: 10.1186/17448069-6-40

Guo, W., Wei, F., Zou, S.-P., Sugiyo, S., Ikeda, T., Meredith, T., et al. (2004). Group I metabotropic glutamate receptor NMDA receptor coupling and signaling cascade mediate spinal dorsal horn NMDA receptor $2 \mathrm{~B}$ tyrosine phosphorylation associated with inflammatory hyperalgesia. J. Neurosci. 24, 9161-9173. doi: 10.1523/jneurosci.3422-04.2004

Guo, W., Yang, J.-L., Imai, S., Zou, S., Li, L.-H., Xu, H. H., et al. (2018) "CCL5/CCR5 signalling in descending circuitry plays a role in bone marrow stromal cell-produced antihyperalgesia," in Abstract, Neuroscience 2018, San Diego, CA, Program No. 2018-S-4337-SfN.

Hargreaves, K. M. (2011). Orofacial pain. Pain 152, S25-32. doi: 10.1016/j.pain. 2010.12.024

Hou, Y.-N., Vlaskovska, M., Cebers, G., Kasakov, L., Liljequist, S., and Terenius, L. (1996). A $\mu$-receptor opioid agonist induces AP-1 and NF-кB transcription factor activity in primary cultures of rat cortical neurons. Neurosci. Lett. 212, 159-162. doi: 10.1016/0304-3940(96)12799-3

Isong, U., Gansky, S. A., and Plesh, O. (2008). Temporomandibular joint and muscle disorder-type pain in U.S. adults: the National Health Interview Survey. J. Orofac. Pain 22, 317-322.

Kou, X., Xu, X., Chen, C., Sanmillan, M. L., Cai, T., Zhou, Y., et al. (2018). The Fas/Fap-1/Cav-1 complex regulates IL-1RA secretion in mesenchymal stem cells to accelerate wound healing. Sci. Transl. Med. 10:eaai8524. doi: 10.1126/scitranslmed.aai8524

Kraus, J., Börner, C., Giannini, E., and Höllt, V. (2003). The role of nuclear factor $\kappa \mathrm{B}$ in tumor necrosis factor-regulated transcription of the human $\mu$-opioid receptor gene. Mol. Pharmacol. 64, 876-884. doi: 10.1124/mol.64.4.876

Ledeboer, A., Gamanos, M., Lai, W., Martin, D., Maier, S. F., Watkins, L. R., et al. (2005). Involvement of spinal cord nuclear factor $\mathrm{\kappa B}$ activation in rat models of proinflammatory cytokine-mediated pain facilitation. Eur. J. Neurosci. 22, 1977-1986. doi: 10.1111/j.1460-9568.2005.04379.x

Liu, A. M., and Wong, Y. H. (2005). $\mu$-opioid receptor-mediated phosphorylation of IкB kinase in human neuroblastoma SH-SY5Y cells. Neurosignals 14 136-142. doi: 10.1159/000086296

Lawrence, T., Gilroy, D. W., Colville-Nash, P. R., and Willoughby, D. A. (2001). Possible new role for NF-кB in the resolution of inflammation. Nat. Med. 7, 1291-1297. doi: 10.1038/nm1201-1291

Long, M., Park, S. G., Strickland, I., Hayden, M. S., and Ghosh, S. (2009). Nuclear factor- $\kappa \mathrm{B}$ modulates regulatory $\mathrm{T}$ cell development by directly regulating expression of Foxp3 transcription factor. Immunity 31, 921-931. doi: 10.1016/j. immuni.2009.09.022

Madge, L. A., and May, M. J. (2011). The NFкB paradox: RelB induces and inhibits gene expression. Cell Cycle. 10, 6-7. doi: 10.4161/cc.10.1.14291

Massa, P. T., Aleyasin, H., Park, D. S., Mao, X., and Barger, S. W. (2006). NFкB in neurons? The uncertainty principle in neurobiology. J. Neurochem. 97, 607-618. doi: 10.1111/j.1471-4159.2006.03810.x

Németh, K., Leelahavanichkul, A., Yuen, P. S., Mayer, B., Parmelee, A., Doi, K., et al. (2009). Bone marrow stromal cells attenuate sepsis via prostaglandin $\mathrm{E}(2)$-dependent reprogramming of host macrophages to increase their interleukin-10 production. Nat. Med. 15, 42-49. doi: 10.1038/nm.1905

Paxinos, G., and Watson, C. (2014). The Rat Brain in Stereotaxic Coordinates. 7th Edn. Amsterdam: Elsevier. 
Pettine, K. A., Murphy, M. B., Suzuki, R. K., and Sand, T. T. (2015). Percutaneous injection of autologous bone marrow concentrate cells significantly reduces lumbar discogenic pain through 12 months. Stem Cells 33, 146-156. doi: 10.1002/stem.1845

Pettine, K., Suzuki, R., Sand, T., and Murphy, M. (2016). Treatment of discogenic back pain with autologous bone marrow concentrate injection with minimum two year follow-up. Int. Orthop. 40, 135-140. doi: 10.1007/s00264-0152886-4

Pilette, C., Detry, B., Guisset, A., Gabriels, J., and Sibille, Y. (2010). Induction of interleukin-10 expression through $\mathrm{F}_{\mathrm{c}} \alpha$ receptor in human monocytes and monocyte-derived dendritic cells: role of p38 MAPKinase. Immunol. Cell Biol. 88, 486-493. doi: 10.1038/icb.2009.120

Pittenger, M. F., Mackay, A. M., Beck, S. C., Jaiswal, R. K., Douglas, R., Mosca, J. D., et al. (1999). Multilineage potential of adult human mesenchymal stem cells. Science 284, 143-147. doi: 10.1126/science.284.5411.143

Ren, K. (1999). An improved method for assessing mechanical allodynia in the rat. Physiol. Behav. 67, 711-716. doi: 10.1016/s0031-9384(99) 00136-5

Ren, K. (in press). Exosomes in perspective: a potential surrogate for stem cell therapy. Odontology

Roh, D. H., Seo, M. S., Choi, H. S., Park, S. B., Han, H. J., Beitz, A. J., et al. (2013). Transplantation of human umbilical cord blood or amniotic epithelial stem cells alleviates mechanical allodynia after spinal cord injury in rats. Cell Transplant. 22, 1577-1590. doi: 10.3727/096368912x659907

Ross-Huot, M. C., Laferrière, A., Khorashadi, M., and Coderre, T. J. (2013). Glycemia-dependent nuclear factor $\kappa \mathrm{B}$ activation contributes to mechanical allodynia in rats with chronic postischemia pain. Anesthesiology 119, 687-697. doi: 10.1097/aln.0b013e318299980c

Sacerdote, P., Niada, S., Franchi, S., Arrigoni, E., Rossi, A., Yenagi, V., et al. (2013). Systemic administration of human adipose-derived stem cells reverts nociceptive hypersensitivity in an experimental model of neuropathy. Stem Cells Dev. 22, 1252-1263. doi: 10.1089/scd.2012.0398

Shen, L. H., Li, Y., Chen, J., Zhang, J., Vanguri, P., Borneman, J., et al. (2006). Intracarotid transplantation of bone marrow stromal cells increases axon-myelin remodeling after stroke. Neuroscience 137, 393-399. doi: 10.1016/j.neuroscience.2005.08.092

Siniscalco, D., Giordano, C., Galderisi, U., Luongo, L., de Novellis, V., Rossi, F., et al. (2011). Long-lasting effects of human mesenchymal stem cell systemic administration on pain-like behaviors, cellular and biomolecular modifications in neuropathic mice. Front. Integr. Neurosci. 5:79. doi: 10.3389/fnint.2011. 00079

Stone, S., Jamison, S., Yue, Y., Durose, W., Schmidt-Ullrich, R., and Lin, W. (2017). NF- $\kappa \mathrm{B}$ activation protects oligodendrocytes against inflammation. J. Neurosci. 37, 9332-9344. doi: 10.1523/JNEUROSCI.1608-17.2017

Tamatani, M., Che, Y. H., Matsuzaki, H., Ogawa, S., Okado, H., Miyake, S., et al. (1999). Tumor necrosis factor induces Bcl-2 and Bcl-x expression through NFKB activation in primary hippocampal neurons. J. Biol. Chem. 274, 8531-8538. doi: 10.1074/jbc.274.13.8531

Vickers, E. R., Karsten, E., Flood, J., and Lilischkis, R. (2014). A preliminary report on stem cell therapy for neuropathic pain in humans. J. Pain Res. 7, 255-263. doi: $10.2147 /$ jpr.s63361

Wagley, Y., Hwang, C. K., Lin, H. Y., Kam, A. F., Law, P. Y., Loh, H. H., et al. (2013). Inhibition of c-Jun NH2-terminal kinase stimulates $\mu$ opioid receptor expression via p38 MAPK-mediated nuclear NF- $\mathrm{B}$ activation in neuronal and non-neuronal cells. Biochim. Biophys. Acta 1833, 1476-1488. doi: 10.1016/j. bbamcr.2013.02.017

Walker, P. A., Harting, M. T., Jimenez, F., Shah, S. K., Pati, S., Dash, P. K., et al. (2010). Direct intrathecal implantation of mesenchymal stromal cells leads to enhanced neuroprotection via an NFкB-mediated increase in interleukin-6 production. Stem Cells Dev. 19, 867-876. doi: 10.1089/scd.2009.0188

Wang, D., and Baldwin, A. S. Jr. (1998). Activation of nuclear factor- $\kappa$ B-dependent transcription by tumor necrosis factor- $\alpha$ is mediated through phosphorylation of RelA/p65 on serine 529. J. Biol. Chem. 273, 29411-29416. doi: 10.1074/jbc. 273.45.29411

Wang, X., Douglas, S. D., Commons, K. G., Pleasure, D. E., Lai, J., Ho, C., et al. (2004). A non-peptide substance P antagonist (CP-96,345) inhibits morphineinduced NF- $\mathrm{KB}$ promoter activation in human NT2-N neurons. J. Neurosci. Res. 75, 544-553. doi: 10.1002/jnr.10873

Watanabe, S., Uchida, K., Nakajima, H., Matsuo, H., Sugita, D., Yoshida, A., et al. (2015). Early transplantation of mesenchymal stem cells after spinal cord injury relieves pain hypersensitivity through suppression of pain-related signaling cascades and reduced inflammatory cell recruitment. Stem Cells 33, 1902-1914. doi: 10.1002/stem.2006

Wei, L. N., and Loh, H. H. (2011). Transcriptional and epigenetic regulation of opioid receptor genes: present and future. Annu. Rev. Pharmacol. Toxicol. 51, 75-97. doi: 10.1146/annurev-pharmtox-010510-100605

Zhou, L., Hu, Y., Li, C., Yan, Y., Ao, L., Yu, B., et al. (2018). Levo-corydalmine alleviates vincristine-induced neuropathic pain in mice by inhibiting an NF-кB-dependent CXCL1/CXCR2 signaling pathway. Neuropharmacology 135, 34-47. doi: 10.1016/j.neuropharm.2018.03.004

Conflict of Interest Statement: The authors declare that the research was conducted in the absence of any commercial or financial relationships that could be construed as a potential conflict of interest.

Copyright (C) 2018 Guo, Imai, Yang, Zou, Li, Xu, Moudgil, Dubner, Wei and Ren. This is an open-access article distributed under the terms of the Creative Commons Attribution License (CC BY). The use, distribution or reproduction in other forums is permitted, provided the original author(s) and the copyright owner(s) are credited and that the original publication in this journal is cited, in accordance with accepted academic practice. No use, distribution or reproduction is permitted which does not comply with these terms. 